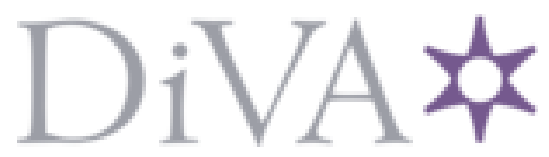

http://www.diva-portal.org

This is the published version of a paper published in IEEE Photonics Technology Letters.

Citation for the original published paper (version of record):

Sudirman, A. (2014)

All-Fiber Optofluidic Component to Combine Light and Fluid.

IEEE Photonics Technology Letters, 26(10): 1031-1033

Access to the published version may require subscription.

N.B. When citing this work, cite the original published paper.

Permanent link to this version:

http://urn.kb.se/resolve?urn=urn:nbn:se:kth:diva-145313 


\title{
All-Fiber Optofluidic Component to Combine Light and Fluid
}

\author{
Aziza Sudirman and Walter Margulis
}

\begin{abstract}
A component is presented that combines light and fluid in an optical fiber arrangement for optofluidics. The component couples light from a standard telecom fiber (STF) to the solid-core of a microstructured fiber, and delivers fluid from a capillary to the holes of the same microstructured fiber. The light is then made to interact longitudinally with the delivered fluid in a hollow-core fiber or capillary. The component is allspliced, hermetic and allows for fluid flow without interrupting the optical beam. Light is brought from the STF to the solid-core/fluid interface with a loss $<0.1 \mathrm{~dB}$.
\end{abstract}

Index Terms-Optical fibers, optical waveguide components, optical fiber coupling, fluidics.

\section{INTRODUCTION}

$\mathbf{M}$ ICROSTRUCTURED fiber and photonic crystal fiber (PCF) are used to a growing extent in optofluidics [1]-[3]. The holes in the fiber provide a natural repository for microfluidic samples, which can be employed for tuning [4], switching [5] and nonlinear optics [6], [7], for instance. The use of fibers in optofluidics is advantageous over planar geometries in two aspects. On one hand, very long length devices can be exploited for enhanced resolution, for example in capillary electrophoresis [8], and on the other, the minute cross-section makes them ideal for in-vivo measurements, e.g., in minimally invasive diagnostics [9], pressure sensing in the body [10] and 3D imaging with fiber optical coherence tomography [11].

Combining light and fluid (liquid or gas) in a small fiber is problematic. The fluid needs to be brought to the fiber without disturbing light coupling or guidance, while light needs to be coupled into the fluid without bubbles or meniscus formation. In a research laboratory, it is possible to use non-ideal bulky cells with optical windows to free-space couple light through lenses and pumps to fill the hollow core with fluid. A more user friendly arrangement, e.g., for future clinical use, should involve only spliced fibers and capillaries with very small dead-volumes. Light would be coupled to conventional fibers, fluids to conventional capillaries and both allowed to interact in a small-volume protected environment. Several techniques have been reported for filling fluid into the holes of microstructured fibers. For instance, blocking cladding holes with a cured

Manuscript received September 27, 2013; revised February 28, 2014; accepted March 5, 2014. Date of publication March 19, 2014; date of current version April 24, 2014. This work was supported in part by the Swedish Research Council and in part by the Linnæus Center of Excellence ADOPT.

The authors are with the Department of Applied Physics, Royal Institute of Technology, Stockholm SE-106 91, Sweden, and also with the Department of Fiber Optics, Acreo Swedish ICT AB, Stockholm SE-164 40, Sweden (e-mail: aziza.sudirman@acreo.se; walter.margulis@acreo.se).

Digital Object Identifier 10.1109/LPT.2014.2312483

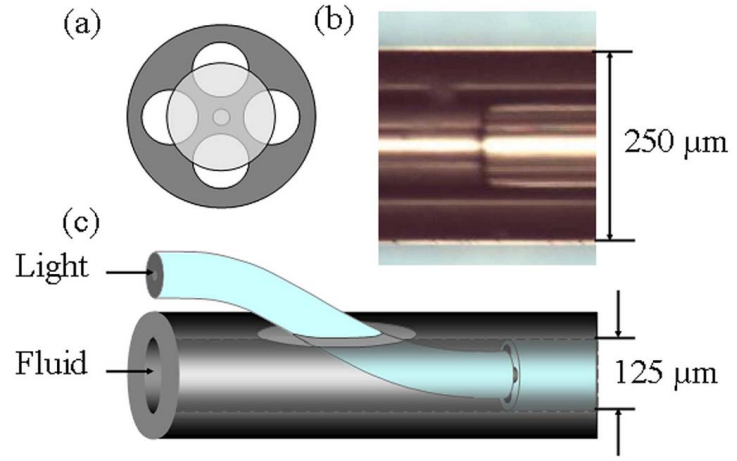

Fig. 1. (a) Schematic illustration of cross section of etched standard telecom fiber spliced to microstructured 4-hole fiber. (b) Microscope image of the spliced 4-hole fiber and etched telecom fiber inside the fluid-delivery capillary. (c) Illustration of the side-polished fluid-delivery capillary.

polymer [12], focused ion beam-milled microchannels on the side of the fiber [13], splicing with a lateral offset [14], using a micropipette [15] and C-shaped fiber for liquid delivery [16] are techniques that allowed for the interaction of a liquidfilled hollow core and light. However, the problem remains of how to collect or administer fluid in a controlled way without disturbing the optical path, providing a hermetic arrangement that prevents harmful fumes and contamination while avoiding the use of bulky components with large dead-volume. This letter presents a method for coupling fluids into the holes of an optical microstructured fiber with basically zero dead-volume, in an all-fiber spliced arrangement, which is hermetic and enables light coupling with minimal loss. It is complemented with an all-spliced arrangement that allows for the interaction of light and fluid.

\section{FABRiCATION PARAMETERS AND ChARACTERIZATION}

The component is made using $125-\mu \mathrm{m}$ diameter silica microstructured fiber and capillaries, preserving the holes of the fiber intact. The microstructured fiber has four $25-\mu \mathrm{m}$ diameter holes (4-hole fiber), and light is guided in the $8-\mu \mathrm{m}$ central core. The component can be used in two directions, for retrieval or delivery of fluid through the holes. In the following, it is assumed that it combines rather than separates light and fluid.

Optical coupling is provided by splicing to the 4-hole fiber a fiber for light delivery of similar core diameter but smaller external diameter. The light-delivery fiber does not cover the entire cross-section of the holes, as schematically illustrated by the light grey cross-section shown in Fig. 1(a). It is manufactured here by stripping the primary coating and then 
etching a 5-cm long end-section of a standard telecom fiber (Corning SMF28) using 40\% hydrofluoric acid. The etched section has outer diameter $50 \mu \mathrm{m}$, while the remaining $\sim 1$-m length of the light-delivery fiber is a piece of SMF28 in its original form. The etched section is cleaved to $\sim 3$-cm length, and the splicing of the matched cores leads to light coupling with $<0.1 \mathrm{~dB}$ loss. Although a narrower $50 \mu \mathrm{m}$ fiber could be used and etching avoided altogether, it is found convenient to use $125 \mu \mathrm{m}$ fiber for optical coupling. Etching many $(>20)$ fibers at once greatly reduces the effort needed per component.

In the first part of the component, the fluid delivered to the 4-hole fiber is contained in an encompassing capillary, as shown in Fig. 1(b), which has inner-diameter $127 \mu \mathrm{m}$ and outer-diameter $250 \mu \mathrm{m}$. A single hole of length $\sim 1 \mathrm{~mm}$ is opened in the fluid-delivery capillary by polishing it from the side, using a rotating drum with attached sand paper. Debris can be minimized by inserting into the hole a piece of sacrificial standard fiber and then removing if after polishing is completed. The etched section of the light-delivery fiber is inserted through this side-hole in the capillary. This makes it possible to bring in the light from the side of the arrangement, as seen in Fig. 1(c), and after splicing, transmit it coaxially to the 4-hole fiber. This is in contrast with previous arrangements, where it is the fluid that is injected through the side-holes.

The etched section of the light-delivery fiber needs to be bent inside the $250 \mu \mathrm{m}$ capillary. The bend is not sharp and is largely determined by the length of the side-hole opened in the capillary. A $1 \mathrm{~mm}$-long hole allows for insertion of the $50 \mu \mathrm{m}$ light-delivery fiber with a shallow angle of $2.9^{\circ}$ (i.e., $\sin ^{-1} 0.05 / 1$ ). For this entrance angle, the fiber first enters in contact with the side-wall of the capillary $3.9 \mathrm{~mm}$ away from the midpoint of the 1-mm hole and the corresponding bend radius is $\sim 7.8 \mathrm{~cm}$, which is considered to be acceptable. Experimentally, the measured loss induced by the bend is $<0.1 \mathrm{~dB}$. To prevent fluid leakage from the polished opening of the fluid-delivery fiber, a suitable adhesive (e.g., UV curing glue Vitralit 2009 F) is used to seal the side-hole entirely.

The etched section of the standard telecom fiber is sufficiently short to be entirely accommodated inside a $(4 \mathrm{~cm})$ conventional splice protector. Outside the splice protector, the light delivery fiber is seen as a standard telecom fiber $(125 \mu \mathrm{m})$. The fluid is delivered coaxially with the arrangement, flowing from the capillary into the non-blocked area of the 4-hole fiber holes. This is also inside the protection sleeve. The small $\sim 2 \mu \mathrm{m}$ gap that exists between the surface of the 4-hole fiber and the inner walls of the capillary needs to be closed to prevent leakage and guarantee hermeticity. This can be done by filling it with a suitable adhesive, or by slightly collapsing the arrangement with a splicer, so that the seal is entirely established by silica.

In some applications the volume contained or conveyed by the $127-\mu \mathrm{m}$ inner diameter capillary may be excessive. One example of such application is an all-fiber nitrobenzene Kerr cell [7], where the liquid is toxic and the volume needed to create a liquid core is very small (150 $\mathrm{nl}$ for a $1-\mathrm{m}$ long component). In such cases, it is possible to reduce the hole size by, for instance, inserting a $125-\mu \mathrm{m}$ outer-diameter capillary with a $\sim 50 \mu \mathrm{m}$ hole into the $250 \mu \mathrm{m}$ capillary, thus
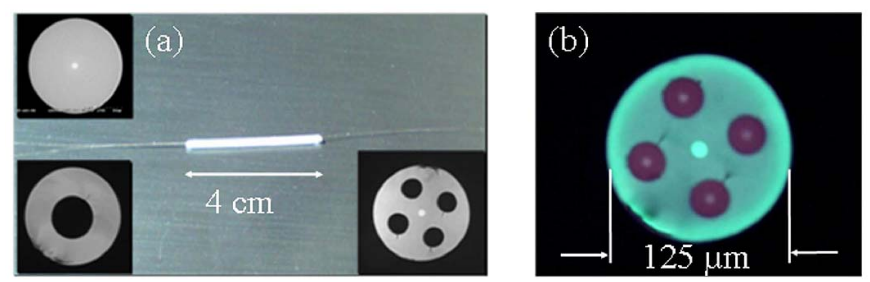

Fig. 2. (a) Image of component for coupling light in the core and fluid in the holes of a microstructured fiber, inside a conventional splice protector. On the left a SEM picture of the light delivery fiber (SMF28) and of a $\sim 50 / 125 \mu \mathrm{m}$ inner/outer diameter capillary. On the right, a SEM picture of the 4-hole fiber. (b) Microscope image of front view of the 4-hole fiber with light and fluid. The core guides green light while the holes are filled with a solution of Rhodamine 6G.

dealing with $<6$ times less volume of fluid. The encompassing $250 \mu \mathrm{m}$ capillary can be made short $(\sim 3 \mathrm{~cm})$. Hence, both the $250 \mu \mathrm{m}$ capillary and the initial section of the smaller $125 \mu \mathrm{m}$ capillary now used for fluid delivery can be housed in the splice protector. The small gap between capillaries can also be closed by weak collapsing in a splicing machine. The resulting arrangement is contained inside a splice protector, which has on one side a 4-hole fiber for light and fluid delivery and has on the other side a standard telecom fiber and a capillary, both with outer-diameter $125 \mu \mathrm{m}$ and protected by the primary coating. This is illustrated in Fig. 2(a).

Fig. 2(b) shows an illuminated central solid-core 4-hole fiber with fluid in the holes. Here, a concentrated solution of Rhodamine 6G (Rh6G) is used for illustration purpose. The light source used here is a tungsten lamp filtered with a $532 \mathrm{~nm}$ interference filter and the fiber facet imaged by a $20 \times$ magnification microscope. To visualize the dye in this picture, the red luminescence is excited from the side by illuminating the 4-hole fiber with $1 \mathrm{~mW}$ light at $405 \mathrm{~nm}$ wavelength from a laser pointer diode. The dye fluorescence light is not guided by the holes because the solution has lower refractive index (1.33) than silica (1.47). Fig. 2(b) illustrates that with the component described above it is possible to couple light in the core and fluid in the side-holes of the microstructured fiber.

\section{LIGHT-FLUID INTERACTION}

Evanescent field interaction between light and the fluid can take place if the holes are sufficiently close to the core. Interaction becomes much more efficient by bringing the light to propagate longitudinally in the fluid (liquid, in the illustration below), as shown in Fig. 3. The 4-hole fiber is spliced to a capillary or hollow-core fiber with relatively large central hole. After splicing, this hole collects the fluid from the 4-hole fiber, and becomes a liquid core in which the light propagates. The fluid-collecting capillary allows for filling the hollow core with liquid and coupling light from a solid-core fiber (with $<0.1 \mathrm{~dB}$ loss), coaxially and again in an all-spliced arrangement [17]. Fig. 3(a) illustrates the entire optofluidic component to combine light and fluid. The component also allows for a continuous fluid flow. The liquid-core section can range from as short as $\sim 20 \mu \mathrm{m}$ to up to meter-long. Fig. 3(b) shows a zoom of a 4-hole solid-core fiber filled with Rh6G dye feeding a 9-mm long liquid-core capillary. No bubbles 
(a)

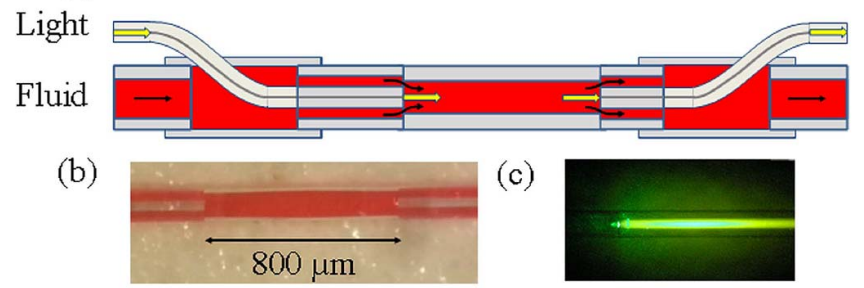

Fig. 3. (a) Schematic illustration of a complete component to combine fluid and light in a spliced arrangement. The yellow and black arrows illustrate light and fluid delivery, respectively. (b) Photo of the section with a dyefilled capillary between two 4-hole fibers. (c) Photo of luminescence resulting from launching pump light into dye, illustrating longitudinal excitation without bubbles or meniscus.

or meniscus are apparent. Fig 3(c) illustrates green laser light coupling from the solid-core of the 4-hole fiber into the central liquid-core in the capillary. Again, the small scattering at the interface implies in the absence of bubbles. The loss by Fresnel reflection between the solid-core and the fluid is $\sim 0.2 \mathrm{~dB}$ for gases and typically $0.01 \mathrm{~dB}$ for liquids (e.g. water). No loss of liquid could be measured by heating an enclosed arrangement to $50{ }^{\circ} \mathrm{C}$, to within $0.02 \%$ uncertainty.

Note that the fluid-collecting capillary could be spliced to a narrower hollow-core capillary or PCF to result in a narrow liquid-core fiber, where high-intensity light can propagate in the fluid (e.g., for nonlinear optics applications). The narrow hollow-core PCF must then act as a waveguide, while the length of the intermediate fluid-collecting capillary should be short $(<50 \mu \mathrm{m})$. The loss of this combined liquid-core section depends very strongly on the refractive index, scattering and absorption of the fluid used and loss of the PCF. The loss in the intermediate capillary in air is measured by increasing the separation between the two solid-core fibers and monitoring the decay in light transmission for a laser at $491 \mathrm{~nm}$ wavelength. By keeping the intermediate capillary length short, e.g., $20 \mu \mathrm{m}$, the loss measured is $6 \pm 3 \%$ and for $50 \mu \mathrm{m}$ the loss is $18 \pm 3 \%$. These values show relatively good agreement with those estimated from a Gaussian beam approximation [18], which works well for a standard fiber core [19].

\section{CONCLUSION}

An all-spliced fiber-based component for combining light and fluid has been presented. The component is rugged and hermetic, the fluid is at all times protected from contamination and the risk of leakage is small (except for gases with very small molecules such as $\mathrm{He}$ and $\mathrm{H}_{2}$, which can diffuse through the silica walls). By inserting the light through a side-hole in a capillary, the end-face of the capillary can be conveniently coupled to a pressure system for liquid or gas delivery with minimal dead-volume. At the same time, an etched fiber can be arranged inside the capillary to provide for low-loss coaxial splicing to a microstructured fiber, for example with 4-holes. The fluid-filled microstructured 4-hole fiber is then spliced to a fluid-combining capillary, where the light from the solid-core is allowed to interact longitudinally with the fluid. This fluidcombining capillary can be cut after a short length and further spliced to a narrow core PCF. The design allows for scaling up to larger diameters and core sizes (e.g., multimode fibers). For easier visualization all illustrations here deal with liquids but the arrangements could also be used in applications where gases are to be injected into the holes of a microstructured fiber.

\section{ACKNOWLEDGMENT}

The authors would like to thank Prof. F. Laurell and Prof. G. Björk (Dept. of Applied Physics, Royal Inst. of Technology, Stockholm) for their support and useful discussions. The authors are also indebted to the personnel of Acreo Fiberlab, who manufactured all fibers and capillaries used in the present work.

\section{REFERENCES}

[1] D. Psaltis, S. R. Quake, and C. H. Yang, "Developing optofluidic technology through the fusion of microfluidics and optics," Nature, vol. 442 , no. 7101, pp. 381-386, 2006.

[2] C. Monat, P. Domachuk, and B. J. Eggleton, "Integrated optofluidics: A new river of light," Nature Photon., vol. 1, pp. 106-114, Feb. 2007.

[3] P. Domachuk and B. J. Eggleton, "Fiber-based optofluidics," Proc. SPIE, vol. 6588 , p. $65880 \mathrm{C}$, May 2007.

[4] P. Mach et al., "Tunable microfluidic optical fiber," Appl. Phys. Lett., vol. 80, no. 23, pp. 4294-4296, 2002.

[5] P. Domachuk, H. C. Nguyen, and B. J. Eggleton, "Transverse probed microfluidic switchable photonic crystal fiber devices," IEEE Photon. Technol. Lett., vol. 16, no. 8, pp. 1900-1902, Aug. 2004.

[6] K. Kieu, L. Schneebeli, R. A. Norwood, and N. Peyghambarian, "Integrated liquid-core optical fibers for ultra-efficient nonlinear liquid photonics," Opt. Express, vol. 20, no. 7, pp. 8148-8154, 2012.

[7] D. Lopez, O. Tarasenko, and W. Margulis, "All-fiber Kerr cell," Opt. Lett., vol. 37, no. 15, pp. 3288-3290, 2012.

[8] M. J. Gordon, X. Huang, S. L. Pentoney, and R. N. Zare, "Capillary electrophoresis," Science, vol. 242, no. 1, pp. 224-228, 1988.

[9] A. Mendez and T. F. Morse, Specialty Optical Fibers Handbook. San Diego, CA, USA: Academic, 2006.

[10] S. Rehman and Å. Claesson, "Specialty optical fibers make surgery less invasive," BioPhotonics, vol. 2, pp. 1-10, Oct. 2011.

[11] B. Lau, R. A. McLaughlin, A. Curatolo, R. W. Kirk, D. K. Gerstmann, and D. D. Sampson, "Imaging true 3D endoscopic anatomy by incorporating magnetic tracking with optical coherence tomography: Proof-ofprinciple for airways," Opt. Express, vol. 18, no. 26, pp. 27173-27180, 2010.

[12] Y. Huang, Y. Xu, and A. Yariv, "Fabrication of functional microstructured optical fibers through selective-filling technique," Appl. Phys. Lett., vol. 85 , no. 22, pp. 5182-5184, 2004.

[13] C. M. B. Cordeiro et al., "Towards practical liquid and gas sensing with photonic crystal fibres: Side access to the fibre microstructure and single-mode liquid-core fiber," Meas. Sci. Technol., vol. 18, no. 10, pp. 3075-3081, 2007.

[14] W. Qian, C. Zhao, Y. Wang, C. C. Chan, S. Liu, and W. Jin, "Partially liquid-filled hollow-core photonic crystal fiber polarizer," Opt. Lett., vol. 36, no. 16, pp. 3296-3298, 2011.

[15] R. M. Gerosa, D. H. Spadoti, C. J. S. de Matos, L. de S. Menezes, and M. A. R. Franco, "Efficient and short-range light coupling to indexmatched liquid-filled hole in a solid-core photonic crystal fiber," Opt. Express, vol. 19, no. 24, pp. 24687-24698, 2011.

[16] S. Hosseinzadeh Kassani, J. Park, M. Park, and K. Oh, "Sensitivity enhancement of in-line chemical sensing device with C-type fiber and photonic crystal fiber," Proc. SPIE, vol. 8237, p. 82371Z, Feb. 2012.

[17] F. Laurell, W. Margulis, V. Pasiskevicius, P. Jelger, Å. Claesson, and A. Engström, "An in-fibre microcavity," in Proc. CLEO, 2007, pp. 1-3, paper $\mathrm{CThO} 2$.

[18] CVI M. Griot, "Gaussian beam propagation," CVI Melles Griot Tech. Guide, vol. 2, no. 2, pp. 2-5, 2009.

[19] A. M. Kowalevicz and F. Bucholtz, "Beam divergence from an SMF-28 optical fiber," Naval Res. Lab., Tech. Rep. NRL/MR/5650-06-8996, 2006. 